\title{
Prevalence of Asthma and Association with Biomass Fuel Use and Other Factors among Adults' Women in Urban Burkina Faso: A Population-Based Cross-Sectional Study
}

\author{
Sana Adama ${ }^{1,4^{*},}$ Meda Nicolas ${ }^{1}$, Ouedraogo Abdoul Risgou ${ }^{1,2}$, Kafando Benoit ${ }^{1}$, Badoum \\ Gisele $^{1,3}$, Bouland Catherine ${ }^{4}$ \\ ${ }^{1}$ Universite Joseph Ki-Zerbo, Ouagadougou, Burkina Faso, West Africa \\ ${ }^{2}$ Centre Hospitalier Universitaire de Tengandogo, Ouagadougou, Burkina Faso, West Africa \\ ${ }^{3}$ Centre Hospitalier Universitaire Yalgado Ouedraogo, Ouagadougou, Burkina Faso, West Africa \\ ${ }^{4}$ Universite Libre de Bruxelles, Ouagadougou, Burkina Faso, West Africa
}

"Corresponding Author: Sana Adama, Universite Joseph Ki-Zerbo, Public Health Laboratory, Ouagadougou, Burkina Faso, Tel: (00226) 704911 62; E-mail: adou_sanette@yahoo.fr

Received: 08 February 2020; Accepted: 18 February 2020; Published: 27 March 2020

Citation: Sana Adama, Meda Nicolas, Ouedraogo Abdoul Risgou, Kafando Benoit, Badoum Gisele, Bouland Catherine. Prevalence of Asthma and Association with Biomass Fuel use and Other Factors among Adults' Women in Urban Burkina Faso: A Population-Based Cross-Sectional Study. Journal of Environmental Science and Public Health 4 (2020): 32-42.

\begin{abstract}
Introduction: Asthma is one of the most significant non-communicable diseases. In 2016, the Global Burden of Disease (GBD) estimated that asthma affects as many as 339 million people worldwide. More than $80 \%$ of asthma deaths occur in low- and middle-income countries. In sub-Saharan Africa, little is known about the burden of asthma.
\end{abstract}

Methods: We did this population-based crosssectional study in Ouagadougou city, Burkina Faso. We randomly selected women above the age of 18 years from 3 neighborhoods in 2 districts. Trained interviewers asked validated questionnaires and administered spirometry to participants. Participants considered with asthma were those who reported wheeze in the past 12 months and/or a physician diagnosis of asthma and/or current use of asthma 
medications and/or have been diagnosed with asthma by the spirometry test. We calculated prevalence of asthma and tested its association with risk factors.

Results: Between March 2017 and September 2018, we interviewed 1705 women, of whom 835 were selected for the spirometry, and among them, 564 provided acceptable spirometry and were analyzed. Mean age was 36 years; none have reported current or former smoking. The prevalence of asthma was $18.18 \%$. The peak of prevalence was observed in the 25-34 years age-group. About 3.28\% reported a physician diagnosis of asthma or use of asthma medications and $16.07 \%$ reported wheeze in the past 12 months. Asthma was associated with biomass use as main cooking fuel (odds ratio 1.33 , 95\% CI 1.02$1.73 ; \mathrm{p}=0.035$ ), having a family history of asthma (2.19, 1.59-3.03; $\mathrm{p}<0.001)$, being in the age group 25-34 years $(1.55,1.05-2.28 ; \mathrm{p}=0.028)$. Living less than 100 meters from a paved road seems to be a protective factor $(0.45,0.34-0.59 ; \mathrm{p}<0.001)$. We didn't find any association between asthma and passive smoking.

Conclusion: Biomass smoke exposure cause damage to women respiratory health. Strategies to improve clean and healthy fuels access are needed.

Keywords: Asthma, prevalence, risk factors, Biomass fuels, Women, sub-Saharan Africa, Burkina Faso

\section{Introduction}

Over the recent years, research has shown an increase in morbidity and mortality associated with noncommunicable diseases (NCDs) in most developing countries [1]. Indeed, in addition to traditional pathologies such as infectious diseases, noncommunicable diseases are increasingly important, in conjunction with socio-cultural changes, urbanization and unhealthy behavioral and lifestyle patterns [2, 3]. Asthma is one of the most significant NCDs. Asthma is a public health priority because it's a cause of substantial burden of disease, including both premature death and reduced quality of life [4]. In 2016, the Global Burden of Disease (GBD) estimated that asthma affects as many as 339 million people worldwide [4]. Asthma occurs in people of all ages in all parts of the world, regardless of the level of development [4]. Globally, asthma is ranked 28th among the leading causes of burden of disease and 27th in low- and middle-income countries (LMICs), as measured by disability adjusted life years (DALYs). In 2016, asthma ranked 23rd (global) and 31st (LMICs) among the leading causes of premature mortality (YLL) [4]. More than $80 \%$ of asthma deaths occur in LMICs [5]. In these countries, it is often an underdiagnosed pathology and insufficiently managed.

Global trends in the burden of asthma are poorly documented. Nearly half of the world's countries have never studied the prevalence of asthma [4]. For many countries in Africa, the latest available data on the prevalence of asthma are those of the world health survey conducted between 2002 and 2003. This study reported an asthma prevalence of $4-8 \%$ in the 18 studied African countries [5]. In Burkina Faso, the same study reported a prevalence rates of $2.02 \%, 2.26$ $\%$, and 5.32, respectively for doctor diagnosed asthma, clinical asthma and wheezing symptoms in adults aged 18 to 45 years [5]. Asthma is a major but 
remediable health problem [4]. Establishing the proportion of the population who have asthma and identifying the risks factors are important for a better management of the disease. To fill the data gap on recent data for asthma prevalence and its risk factors in Burkina Faso, we aimed to conduct a populationbased survey in urban Burkina Faso, especially in Ouagadougou.

\section{Materials and Methods}

\subsection{Type of study}

This study was a cross-sectional population-based survey carried out between March 2017 and September 2018, in Ouagadougou city, Burkina Faso.

\subsection{Field and study population}

Ouagadougou is the largest city and the capital of Burkina Faso. According to the estimates, in 2012, the population of Ouagadougou is about 1915102 inhabitants, distributed in 55 neighborhoods and 12 districts [6]. The survey was conducted in three neighborhoods located in two districts: one neighborhood (Tanghin) of the district 14 and two neighborhoods (Kilwin and Tampouy) of the district 15 of Ouagadougou. These districts are those covered by the Ecosanté project's intervention. The selected neighborhoods are residential locality fairly representative of the other districts of Ouagadougou regarding socio-economic characteristics and models of space occupation. Two of their 3 neighbourhoods host the Ouagadougou Population Observatory (OPO), which also include 3 informal neighborhoods (also called spontaneous neighbourhoods). In 2012, there were 67,394 inhabitants in sector $15,71,628$ in habitants in sector 16 and 64,694 inhabitants in sector 17 with $50.23 \%, 51.49 \%$ and $50.28 \%$ women

Journal of Environmental Science and Public Health respectively [6].

\subsection{Sample and inclusion criteria}

With the tool statcalc of EpiInfo version 7.2.2.2 for calculating sample size for population survey or descriptive study, based on an expected prevalence of $5.32 \%$ [5], with $2 \%$ acceptable margin for error and $99.9 \%$ confidence level, allowing for a $10 \%$ refusal to participate to the study, 1490 subjects were needed to be involved in the survey. A list of the households in the concerned neighborhoods was unavailable. The approach used for the sampling was the itineraries method [7]. Households to be included should have a woman or a girl of at least 18 years old and be living in the neighborhood for at least 02 years. In each concession (extended family house or multifamily home), only one household was selected. Then, the woman mainly in charge of cooking in the household was interviewed.

\subsection{Definition of asthma}

There is no gold standard test for asthma diagnosis [8]. In developing countries, a strict definition of asthma based solely on doctor diagnosis is not suitable to classify individuals with asthma. Since diagnosis and the availability of treatment may be challenging in resource-poor countries, a broader definition which includes respiratory symptoms, in addition to doctor diagnosis and treatment received may yield a higher sensitivity in identifying individuals with asthma [5]. Similarly, to the methodology used in the World Health Survey (WHS), we estimated the prevalence of asthma using three definitions of asthma: 1- self-reported physician diagnosed asthma which is based on the question "Have you ever been diagnosed with asthma?" and/or 
treated asthma based on a positive response in either of two questions "Have you ever been treated for asthma“, 2- based on self-reported physician diagnosed asthma, treated asthma and/or symptoms of asthma based on a positive response to "During the last 12 months have you experienced attacks of wheezing or whistling breath?" [5]. We considered a third definition that include doctor diagnosed asthma and or treated asthma, symptoms of asthma and/or spirometry-diagnosed asthma.

\subsection{Data collection techniques}

The study was conducted in 2 steps.

In the first step we used a standardized questionnaire. The energy choices for cooking, socio-cultural, economic, demographic and personal characteristics of the woman participant were collected in this questionnaire. Objective respiratory health data were also collected. Respiratory health information collected from the participant concerned the symptoms of obstructive respiratory diseases such as COPD including chronic bronchitis (CB), and asthma. Concerning asthma, the following questions were asked to the woman:

1. Do you cough several times most days?

2. Have you had wheezing or whistling in your chest at any time in the last 12 months?

3. If you make an intense physical effort (run, or climb stairs fast) do you ever cough?

4. If you make an intense physical effort (run, or climb stairs fast) do you ever wheeze?

5. If you make an intense physical effort (run, or climb stairs fast) do you ever get tight in the chest?
6. If you make an intense physical effort (run, or climb stairs fast) do you ever get difficulty with breathing?

7. Have you woken up by an attack of shortness of breath at any time in the last 12 months?

8. Have you been woken by an attack of coughing at any time in the last 12 months?

9. Have you woken up with a feeling of tightness in your chest at any time in the last 12 months?

10. A member of your family had been diagnosed with asthma (father, mother, sister, brother, daughter, son)?

11. Have you ever been told by a health worker that you have had asthma, or have you ever been treated for asthma?

12. Have you had an attack of asthma in the last 12 months?

In the second step, all the participant women who answer in the affirmative (yes) to at least three of the first ten questions listed above, or those who gave minimum three positive response to the GOLD questionnaire [9], were invited to perform spirometry test. portable spirometer "spirobank II advanced" was used. Spirometry was performed seated and according to the American Thoracic Society (ATS) criteria, [10] by trained physicians. The expected values were calculated based on data from the National Health and Nutrition Examination Survey (NHANES III). At least three measures had to be carried out for each participant and the highest results were considered. When the ratio forced expiratory volume in the 1 st second to forced vital capacity is less than 0.70 (FEV1/FVC <0.70), 200 micrograms of salbutamol was administered [11], always with the consent of the participant. In this case, post-bronchodilation 
spirometry is performed at least 10 minutes after bronchodilator administration. The results were reviewed by a pulmonologist located in the university hospital Yalgado Ouedraogo (CHUYO).

\subsection{Data analysis}

Data were encoded using Epi-Data software. The sample description and analysis were done on Stata software 13.0, [12]. The dependent variable is asthma. Participants considered with asthma were those who reported wheeze in the past 12 months and/or a physician diagnosis of asthma and/or a use of asthma medications and/or have been diagnosed with asthma by our spirometry test. We consider those who software 13.0, [12]. The dependent variable is asthma. Participants considered with asthma were those who reported wheeze in the past 12 months and/or a physician diagnosis of asthma and/or a use of asthma medications and/or have been diagnosed with asthma by our spirometry test. We consider those who answered "I don't know" to the wheezing question as not have experienced wheezing. The independent variables analyzed were the main cooking fuel (the main fuel used by the woman at home for cooking), age (participant age in years, at the time of the survey), the exposition to road traffic pollution (residence less than 100 meters from a paved road), cigarette smoking status (active smoking and passive smoking), family history of asthma (father, mother, sister, brother, daughter, son). Passive smoking means the exposure to smoke from other people's cigarettes, cigars, or pipes, at home and/or at the workplace. Some variables have been dichotomized as follows: the main fuel used was dichotomized as biomass vs. non-biomass. The variable passive smoking was divided into 2 groups: no for those who are not exposed, yes for those exposed at home and/or at work. The participant age has been grouped into 6 age-classes: 18-24, 25-34, 35-44, 45-54, 55-64, 65+ (65 and more). The other variables were dichotomized as yes and no. We used conventional statistics to describe the sample. Independent variables have been described using frequencies. Prevalence of asthma was estimated and presented as number and percentage. The association between the independent variables and the variables of interest was assessed using Pearson's chi-square test. In the case of the nonapplicability of the chi-square test (expected <5), Fischer's exact test was used. We performed bivariate analysis to study the associations between each independent variable and asthma. After that, logistic regression was applied to identify the risk factors for asthma. All variables that were associated with a Pvalue $<0.1$ in bivariate analysis were then considered or inclusion in a logistic regression model. A p-value $<0.05$ is considered as significant.

\section{Results}

At the end of the survey 1705 participants completed the questionnaire. Among them, 835 participants met the selection criteria to undergo spirometry. Finally, 564 women completed a valid test. Two hundred and twenty-four participants (224) did not perform the lung function test, either because they refused, or they were unavailable, very busy or had moved or were travelling during the study period. Forty-seven (47) invalid tests were not considered.

\subsection{Descriptive analysis of variables}

3.1.1 Socio-demographic characteristics: The surveyed population median age was 36 years (18 to 
85 years). None of the respondents reported current or former smoking. Some characteristics of the population was reported in the (Table 1). The detailed socio-demographic characteristics have been published elsewhere [13].

3.1.2 Prevalence: The overall prevalence of asthma was $18.18 \%$ (310). Fifty-six (56) women (3.28\%) have reported a medical diagnosis of asthma and/or being treated for asthma, and among them 91.07\%
(51) reported having had an asthma attack in the last 12 months prior to the survey, among 1,705 women, the prevalence of women who reported wheezing in the last 12 months prior to the study was $16.07 \%$ (274). Fourteen women $(0.82 \%)$ were diagnosed with asthma after spirometry with bronchodilator administration (200ug of salbutamol). The highest prevalence of overall asthma by age group was observed in the women aged above 64 years (Table 2).

\begin{tabular}{|c|c|c|}
\hline Variables & Effective & Percentage (\%) \\
\hline \multicolumn{3}{|l|}{ Age in years } \\
\hline $18-24$ & 324 & 19.00 \\
\hline $25-34$ & 586 & 34.37 \\
\hline $35-44$ & 391 & 22.93 \\
\hline $45-54$ & 241 & 14.13 \\
\hline $55-64$ & 118 & 6.92 \\
\hline $65+$ & 45 & 2.64 \\
\hline \multicolumn{3}{|c|}{ Active smoking } \\
\hline Yes & 0 & 0 \\
\hline No & 1705 & 100 \\
\hline \multicolumn{3}{|c|}{ Passive smoking } \\
\hline Yes & 619 & 36.31 \\
\hline No & 1086 & 63.69 \\
\hline \multicolumn{3}{|c|}{ Main cooking fuel } \\
\hline Biomass & 1015 & 59.53 \\
\hline Non-biomass & 690 & 40.47 \\
\hline \multicolumn{3}{|c|}{ Family history of asthma } \\
\hline Yes & 226 & 13.26 \\
\hline No & 1479 & 86.74 \\
\hline \multicolumn{3}{|c|}{ Proximity to high traffic road } \\
\hline Yes & 722 & 42.35 \\
\hline No & 983 & 57.65 \\
\hline
\end{tabular}

Table 1: Characteristics of the study subjects ( $N$ 1705). 


\begin{tabular}{|c|c|c|c|}
\hline \multirow[t]{2}{*}{ Variables } & \multirow[t]{2}{*}{$\mathbf{n} / \mathbf{N}$} & \multicolumn{2}{|c|}{ Prevalence } \\
\hline & & $\%$ & $95 \%$ CI \\
\hline Overall & $310 / 1705$ & 18.18 & $16.35-20.01$ \\
\hline \multicolumn{4}{|l|}{ Age in years } \\
\hline $18-24$ & $42 / 324$ & 12.96 & $9.72-17.09$ \\
\hline $25-34$ & $118 / 586$ & 20.14 & $17.08-23.58$ \\
\hline $35-44$ & $68 / 391$ & 17.39 & $13.94-21.48$ \\
\hline $45-54$ & $52 / 241$ & 21.58 & $16.82-27.23$ \\
\hline $55-64$ & $19 / 118$ & 16.10 & $10.49-23.91$ \\
\hline $65+$ & $11 / 45$ & 24.44 & $13.98-39.16$ \\
\hline \multicolumn{4}{|c|}{ Passive smoking } \\
\hline Yes & $119 / 619$ & 19.22 & $16.28-22.49$ \\
\hline No & $191 / 1086$ & 17.59 & $15.45-19.99$ \\
\hline \multicolumn{4}{|c|}{ Main cooking fuel } \\
\hline Biomass & $201 / 1015$ & 19.80 & $17.46-22.37$ \\
\hline Non-biomass & $109 / 690$ & 15.80 & $13.26-18.72$ \\
\hline \multicolumn{4}{|c|}{ Family history of asthma } \\
\hline Yes & $68 / 226$ & 30.09 & $24.45-36.40$ \\
\hline No & $242 / 1479$ & 16.36 & $14.56-18.34$ \\
\hline \multicolumn{4}{|c|}{ Proximity to paved road } \\
\hline Yes & $85 / 722$ & 11.77 & $9.61-14.34$ \\
\hline No & $225 / 983$ & 22.89 & $20.37-25.62$ \\
\hline
\end{tabular}

Table 2: Prevalence of asthma by age group, passive smoking status, cooking fuel, family history of asthma and road exposure.

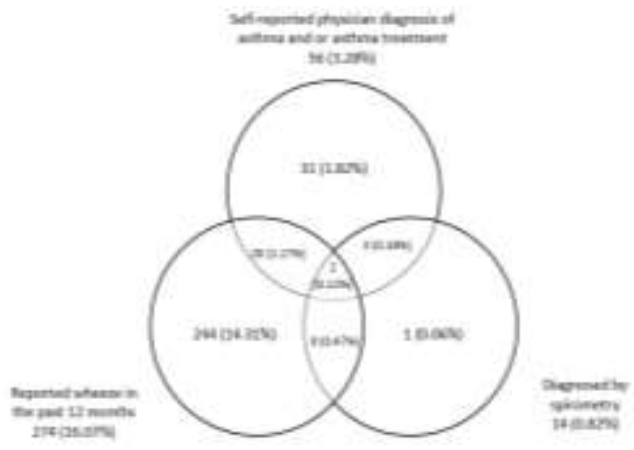

Figure 1: A venn-diagram showing asthma prevalence by three diagnosis criteria and overlap between them [8]. 
The (Figure 1) shows the prevalence of asthma by three diagnosis criteria (taking account of the definitions used in the WHS) and overlap between them.

\begin{tabular}{|c|c|c|c|c|c|c|}
\hline Factors & $\begin{array}{l}\text { With } \\
\text { asthma } \\
\text { n (\%) }\end{array}$ & $\begin{array}{l}\text { Without } \\
\text { Asthma } \\
\text { n }(\%)\end{array}$ & $\begin{array}{l}\text { Crude estimates } \\
\text { Odds Ratio }(95 \% \\
\text { CI) }\end{array}$ & P-value & $\begin{array}{l}\text { Adjusted } \\
\text { estimates } \\
\text { Odds Ratio (95\% } \\
\text { CI) }\end{array}$ & P-value \\
\hline \multicolumn{7}{|l|}{ Age in years } \\
\hline $18-24$ & $42(13.55)$ & $282(20.22)$ & 1 (reference) & & 1(reference) & \\
\hline $25-34$ & $118(38.06)$ & $468(33.55)$ & $1.69(1.15-2.48)$ & 0.007 & $1.55(1.05-2.28)$ & 0.028 \\
\hline $35-44$ & $68(21.94)$ & $323(23.15)$ & $1.41(0.93-2.14)$ & 0.103 & NS & - \\
\hline $45-54$ & $52(16.77)$ & $189(13.55)$ & $1.85(1.18-2.89)$ & 0.007 & NS & - \\
\hline $55-64$ & $19(6.13)$ & $99(7.10)$ & $1.29(0.71-2.32)$ & 0.398 & NS & - \\
\hline $65+$ & $11(3.55)$ & $34(2.44)$ & $2.17(1.02-4.61)$ & 0.044 & NS & - \\
\hline \multicolumn{7}{|c|}{ Passive smoking } \\
\hline No & $191(61.61)$ & $894(64.09)$ & 1 (reference) & & - & \\
\hline Yes & $119(38.39)$ & $501(35.91)$ & $1.11(0.86-1.43)$ & 0.413 & - & - \\
\hline \multicolumn{7}{|c|}{ Main cooking fuel } \\
\hline Non-biomass & $109(35.16)$ & $581(41.65)$ & 1 (reference) & & 1 (reference) & \\
\hline Biomass & $201(64.84)$ & $814(58.35)$ & $1.32(1.02-1.70)$ & 0.036 & $1.33(1.02-1.73)$ & 0.035 \\
\hline \multicolumn{7}{|c|}{ Family history of asthma } \\
\hline No & $242(78.06)$ & $1237(88.67)$ & 1 (reference) & & 1 (reference) & \\
\hline Yes & $68(21.94)$ & $158(11.33)$ & $2.20(1.60-3.02)$ & $<0.001$ & $2.19(1.59-3.03)$ & $<0.001$ \\
\hline \multicolumn{7}{|c|}{ Proximity to high traffic road } \\
\hline No & $225(72.58)$ & $758(54.34)$ & 1 (reference) & & 1 (reference) & \\
\hline Yes & $85(27.42)$ & 637 (45.66) & $0.45(0.34-0.59)$ & $<0.001$ & $0.45(0.34-0.59)$ & $<0.001$ \\
\hline
\end{tabular}

Hosmer-Lemeshow chi2(8) = 3.96; Prob > chi2 = 0.8603; NS: not significant.

Table 3: Factors associated with asthma: univariate and multivariate analysis.

3.1.3 Bivariate and multivariate analysis: In the bivariate analysis, asthma was significantly associated with the participant age, the main cooking fuel used for cooking, the history of asthma in the family and proximity of the home with a paved road (see Table $3)$. Asthma prevalence was statistically higher for 25 -
34, 45-54 and 65+ age groups when compared to women aged between 18-24 years. Asthma is also more prevalent among biomass users when compared to non-biomass users. It is also the case of those who reported asthma diagnosis among a member of their family. Living less than 100 meters from a paved road 
seems to be a protective factor $(\mathrm{OR}=0.45$, CI: 0.34 0.59 ). We didn't find any association between passive smoking and asthma. The multivariate analysis showed a significant association between asthma and the age group 25-34 years, biomass use as main cooking fuel, having a family member with asthma and living more than $100 \mathrm{~m}$ from paved road (see Table 3).

\section{Discussion}

The prevalence of self-reported physician diagnosed and/or currently treated asthma was 3.28\% (56 women). This prevalence is higher than that recorded in the country during the WHO survey, conducted between 2002 and 2003 [5]. Adeloye in his systematic analysis of the prevalence of asthma for Africa for 1990, 2000, and 2010, noted an increasing prevalence of asthma in Africa over the past two decades [1]. But our finding can be explained by a diagnosis improvement (awareness, information, availability). The overall prevalence of asthma in the present study (18.18\%) was higher in comparison with the prevalence reported in other studies $[5,8,14,15]$. The prevalence estimated was much higher than that estimated by Kirenga in Uganda (11.4\%) [8], To in 70 countries (8.6\%) [5], Tarraf in five middle eastern countries (6.4\%) [14] and Agrawal in India (1.9\%) [15]. According to World Health Organization (2012), the overall prevalence of asthma in Burkina Faso was $5.32 \%$ between 2002-2003 [5]. The difference of prevalence observed between the present study and other studies could be due to the characteristics of the population included in our study. We have selected only women who reported being in charge of the cooking in their home. Hence, the difference could be due to smoke exposure during cooking activities.
Indeed, our study and several previous studies have reported that biomass smoke exposure was associated with asthma $[8,15]$. We found that about $60 \%$ of our studied women reported the use of biomass-based fuel as primary fuel for cooking. But, more than $80 \%$ of the women rely on firewood or charcoal in the cooking in second or third order, in combination with other fuel like liquefied petroleum gas (LPG).

Also, some studies have highlighted that asthma was more common among urban people compared to rural ones and also among women in comparison to men $[1,8,14,15]$. Interestingly, we have found inverse association between road exposure and asthma. Asthma was more prevalent in women who reported live more than 100 meters from a paved road as compared to those who live less than 100 meters from a paved road, with a statistically significant difference, even after adjustment (OR: 0.45, CI (0.340.59)). The exact reason behind this is unknown and yet to be explored. Most of the people living far from paved road have their home located close unpaved roads. We speculate that, on unpaved roads, dust emanating from vehicle traffic (cars, numerous motorcycles, bicycles) and from the wind contribute to ambient particulate matter pollution-exposure. Dust exposure would cause health disorders [16]. Road paving reduces air pollution caused by dust particles released into the air [17]. The peak of asthma was observed in the 65+ age group, followed by the 45-54. The age group 25-34 was found to be an important risk factor for asthma. Other studies reported that asthma prevalence increase with age with statistically significant difference [14, 15]. Similarly to our finding, Kirenga reported an association between asthma and family history of asthma (AOR 2.90 
(1.98-4.22)) [8].Other studies have suggested that having a family history of asthma may increase the risk to develop asthma $[18,19]$.

\section{Conclusion}

Asthma is common in Ouagadougou. Asthma is prevalent in Ouagadougou but largely undiagnosed and undertreated. Biomass smoke exposure is a preventable risk factor independently associated with the disease. Awareness of asthma and access to clean and healthy fuels strategies must be improved.

\section{Acknowledgements}

Adama Sana was supported by a $\mathrm{PhD}$ studentship granted by the International Development Research Centre (IRDC) via the CoPEH-WCA, and the ULB cooperation funds. Acknowledgements to ULB and to Health Inter Network Access to Research Initiative (HINARI) team for providing us opportunities to access many scientific publications. We are thankful to all the participants of the study for their availability, and to all the participating associations and their affiliates for their support.

\section{Conflict of Interest}

We declare no competing interests.

\section{References}

1. Adeloye D, Chan KY, Rudan I, et al. An estimate of asthma prevalence in Africa: a systematic analysis. Croatian medical journal 54 (2013): 519-531.

2. Miranda JJ, Kinra S, Casas JP, et al. Noncommunicable diseases in low- and middleincome countries: context, determinants and

Journal of Environmental Science and Public Health health policy. Tropical medicine \& international health : TM and IH 13 (2008): 1225-1234.

3. World Health Organization (WHO). Global Action Plan for the Prevention and Control of Noncommunicable Diseases 2013-2020 (2013).

4. Global Asthma Network. The Global Asthma Report. Retrieved from Auckland, New Zealand (2018).

5. To T, Stanojevic S, Moores G, et al. Global asthma prevalence in adults: findings from the cross-sectional world health survey. BMC Public Health 12 (2012): 204.

6. Mairie de Ouagadougou. Analyse synthétique de l'énumération de la population de Ouagadougou 2012. Ouagadougou: Mairie de Ouagadougou (2012): 20.

7. Sana A, Meda N, Badoum G, et al. Primary Cooking Fuel Choice and Respiratory Health Outcomes among Women in Charge of Household Cooking in Ouagadougou, Burkina Faso: Cross-Sectional Study. International journal of environmental research and public health 16 (2019): 1040.

8. Kirenga BJ, de Jong C, Katagira $\mathrm{W}$, et al. Prevalence and factors associated with asthma among adolescents and adults in Uganda: a general population based survey. BMC Public Health 19 (2019): 227.

9. Global initiative for Chronic Obstructive Lung Disease. "Could it be COPD?" Questionnaire (2004).

10. Standardization of Spirometry. American Thoracic Society. Am J Respir Crit Care Med 152 (1995): 1107-1136.

11. Sichletidis L, Spyratos D, Papaioannou M, et al. A combination of the IPAG questionnaire and 
PiKo-6® flow meter is a valuable screening tool for COPD in the primary care setting. Prim Care Respir J 20 (2011): 184-189.

12. Stata Corporation. Stata 13.0 (Version 13.0) [Sofware]. College Station City, TX: StataCorp LP., USA: StataCorp (2015).

13. Sana A, Kafando B, Dramaix $M$, et al. Household energy choice for domestic cooking: distribution and factors influencing cooking fuel preference in Ouagadougou. Environmental Science and Pollution Research (2020): 1-9.

14. Tarraf H, Aydin O, Mungan D, et al. Prevalence of asthma among the adult general population of five Middle Eastern countries: results of the SNAPSHOT program. BMC Pulm Med 18 (2018): 68.

15. Agrawal S, Pearce N, Ebrahim S. Prevalence and risk factors for self-reported asthma in an adult indian population: a cross-sectional survey. Int J Tuberc Lung Dis 17 (2013): 275-282.

16. Goudie AS. Desert dust and human health disorders. Environment International 63 (2014): 101-113.

17. U.S. Department of transportation. MultiPollutant Emissions Benefits of Transportation Strategies-FHWA.

18. Burke W, Fesinmeyer M, Reed K, et al. Family history as a predictor of asthma risk. Am J Prev Med 24 (2003): 160-169.

19. Liu T, Valdez R, Yoon PW, et al. The association between family history of asthma and the prevalence of asthma among US adults: National Health and Nutrition Examination Survey, Genet Med 11 (2009): 323-328.

This article is an open access article distributed under the terms and conditions of the Creative Commons Attribution (CC-BY) license 4.0 